\title{
Using magnetic ray tracing to reproduce the Sun's cosmic-ray shadow as seen by IceCube
}

\author{
Jens Kleimann, ${ }^{a, *}$ Frederik Tenholt, ${ }^{a}$ Niklas Döpper, ${ }^{a}$ Julia Becker Tjus, ${ }^{a}$ Horst \\ Fichtner $^{a}$ and Paolo Desiati ${ }^{b}$ \\ ${ }^{a}$ Theoretische Physik IV, Ruhr-Universität Bochum, 44780 Bochum, Germany \\ ${ }^{b}$ Dept. of Physics and Wisconsin IceCube Particle Astrophysics Center, \\ University of Wisconsin-Madison, Madison, WI 53706, USA \\ E-mail: jk@tp4.rub.de, ftenholt@tp4.rub.de, niklas.doepper@rub.de, \\ julia.tjus@rub.de, hf@tp4.rub.de, paolo.desiati@icecube.wisc.edu
}

The cosmic-ray Sun shadow is caused by high-energy charged cosmic rays (CRs) being blocked and deflected by the Sun and its magnetic field, thereby modulating the resulting shadow in both size and shape. Recent Sun shadow observations by ground-based particle observatories have established a novel and potentially fruitful link between solar physics and high-energy particle astrophysics. Most notably, the shadow's size and depth was recently shown to correlate with the 11-year solar cycle. This talk addresses the observational situation, the general setup and implementation of our group's Sun shadow simulations, test cases, and actual simulations of increasing complexity. Based on extrapolations from magnetograms, we create artificial shadow images by numerically computing trajectories of charged CRs in the coronal magnetic field for the energy range of 5-316 TeV and for various mass numbers and typically measured CR spectra, and analyze these images in comparison to data from the IceCube neutrino observatory. We confirm the observationally established correlation between the magnitude of the shadowing effect and both the mean sunspot number and the polarity of the magnetic field during the solar cycle. Contrary to previous findings, a non-monotonous dependence on energy during solar minimum is identified and modeled using a simplified (dipolar) configuration for the coronal magnetic field.

$37^{\text {th }}$ International Cosmic Ray Conference (ICRC 2021)

July 12 th - 23rd, 2021

Online - Berlin, Germany

\footnotetext{
*Presenter
} 


\section{Introduction}

Extended celestial objects, most notably the Sun and the Moon, can block incoming cosmic rays (CRs), causing a localized decrease of intensity known as the CR shadow of the body in question. This phenomenon was observed as early as 1993 [1], and has more recently also been identified in CR intensity maps created using data from various observational facilities (see [2] and references therein) including IceCube, see left panel of Figure 1. While the observed Moon shadow is consistent with simple geometrical shielding by a solid, Moon-sized sphere, the Sun shadow shows waxing and waning on a timescale of about eleven years. This can be further quantified as shown in the right panel of Figure 1, indicating that shallow/weak shadows correlate well with proxies of high solar activity, such as sunspot number. This has been tentatively explained by arguing that a strong coronal magnetic field will divert many particles towards Earth, thereby brightening the shadow region. However, the same line of reasoning could also be used to argue that many particles will likewise get deflected away from Earth. The net effect is therefore yet unclear, and the correlation indicated in Figure 1 thus motivates more in-depth studies and simulations.

Furthermore, not only the absolute field strength, but also the qualitative structure of the coronal magnetic field varies throughout the solar cycle, namely from a dipolar field being dragged out radially by the solar wind during times of solar minimum, towards a highly irregular, asymmetric field of mixed polarity during solar maximum. Using full-orbit particle simulations, it will be demonstrated in this paper that the latter effect does indeed exert a crucial influence on the resulting shadow size/depth.

\section{Simulation method and Tests}

Because travel times of CRs through the solar corona amount to mere seconds, the Sun's gravity can safely be ignored when determining the particles' dynamics. Likewise, the motional electric
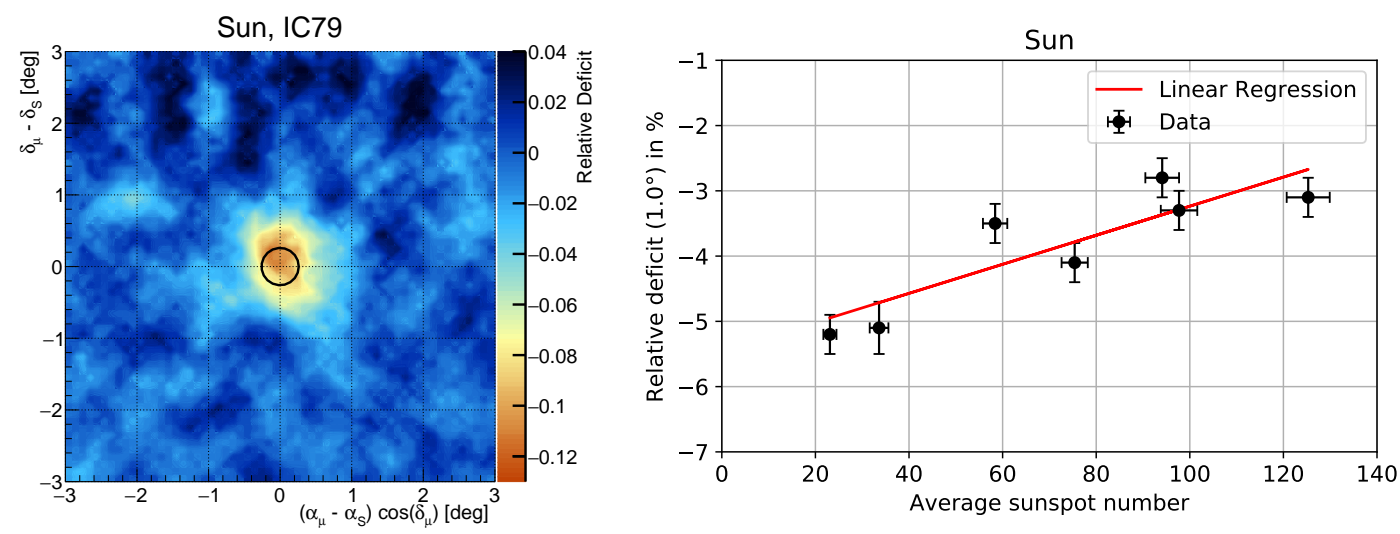

Figure 1: Left: the CR Sun shadow as observed by IceCube [3], shown as a sky map of relative deficit (decrease of intensity relative to the mean background level). The black circle marks the solar disk. Right: correlation between the integrated deficit (normalized to the area of the solar disk) as observed by IceCube in seasons 2010/11-2016/17 vs. sunspot number, with each data point representing a different year. Taken from [4]. 
field $\vec{E}=-\vec{v}_{\mathrm{sw}} \times \vec{B}$ induced by the velocity $\vec{v}_{\mathrm{sw}}$ of the solar wind plasma is of order $\left|\vec{v}_{\mathrm{sw}}\right| / c \sim 10^{-4}$, and can therefore be neglected as well. The trajectory $t \mapsto \vec{r}(t)$ of a CR particle (rest mass $m$, charge $q=Z e$ ) in a magnetic field $\vec{B}$ is therefore governed by the system

$$
\frac{\mathrm{d} \vec{r}}{\mathrm{~d} t}=\vec{v} \quad \wedge \quad \frac{\mathrm{d}(\gamma m \vec{v})}{\mathrm{d} t}=q \vec{v} \times \vec{B}
$$

or, in dimensionless form,

$$
\frac{\mathrm{d} \vec{r}^{\prime}}{\mathrm{d} t^{\prime}}=\vec{v}^{\prime} \quad \wedge \quad \frac{\mathrm{d} \vec{v}^{\prime}}{\mathrm{d} t^{\prime}}=\eta \vec{v}^{\prime} \times \vec{B}^{\prime}
$$

with normalization constants $L_{0}=R_{\odot}$ (the solar radius), $v_{0}=c$, and $t_{0}=R_{\odot} / c$. This results in

$$
\eta=\frac{Z e B_{0} R_{\odot}}{\gamma m c} \approx 209 Z \frac{B_{0}[\mathrm{mT}]}{E[\mathrm{TeV}]}=209 \frac{B_{0}[\mathrm{mT}]}{P[\mathrm{TV}]}
$$

as the only free parameter, in which rigidity is to be defined as $P:=\left(\gamma m c^{2}\right) / q$ rather than just $(\gamma m c) / q$. Note that the Lorentz factor $\gamma=\gamma(|\vec{v}|)$ can be treated as a constant since the Lorentz force cannot change the magnitude of $\vec{v}$.

Equation (2) can be integrated numerically in a multitude of ways, with the Boris push [5] being among the most appropriate ones since it merely rotates $\vec{v}$ but, by construction, leaves $|\vec{v}|$ unchanged, along with the particle's energy.

In our setup, we place the center of the Sun at the origin of a Cartesian coordinate system with unit vectors $\left\{\vec{e}_{x}, \vec{e}_{y}, \vec{e}_{z}\right\}$ and the axis of solar rotation pointing along $+\vec{e}_{z}$. The terrestrial observer is located at $\vec{r}_{0}=d_{0} \vec{e}_{x}$ at a distance $d_{0}=1 \mathrm{au} / R_{\odot} \approx 215$, with all distances measured in units of $R_{\odot}$. The observer's quadratic viewing window is oriented normal to $\vec{e}_{x}$ and located at $-\left(d_{0}-5\right) \vec{e}_{x}$, spanning a $3^{\circ} \times 3^{\circ}$ field of view centered on the Sun. The view window is then split into a grid of (typically) $100 \times 100$ pixels, and virtual particles are launched from the observer through each pixel and towards the coronal region behind it. Once a particle enters a magnetized region, its trajectory is integrated using Equation (2) until it either intersects the photosphere (i.e. the unit sphere $r=1$ ) or escapes into an unmagnetized region at larger heliocentric distance. The corresponding pixel is then colored depending on the particle's fate as either, say, black (0) for particles hitting the Sun, or white (1) for those avoiding it, resulting in a binary (black/white, 0/1) shadow map.

While actual particles coming in effectively from infinity towards the detector would of course traverse the trajectory in the opposite direction, only a very small number of them will pass through the viewing window and contribute to the observed image. But since a joint inversion of both $t$ and $q$ leaves the dynamics of Equation (2) unchanged, the above procedure can be made fully equivalent to the actual direction of propagation, provided that the particle's charge is also reversed. For this reason, our procedure of back-tracking anti-particles, which closely follows the concept of optical ray tracing in inhomogeneous media (see, e.g. [6, 7]), allows for very efficient image generation by a priori elimination of all non-contributing trajectories.

\section{Test cases}

As usual with numerical simulations, verifying the correct operation of the code and the physical meaningfulness of results thus obtained is both vital and nontrivial. Even for comparatively basic magnetic field configurations, the resulting family of nonlinear trajectories is typically not accessible 
to analytical solutions against which results could be gauged. In order to familiarize ourselves with the relationship between a given three-dimensional field and the resulting shadow images, it was found instructive to not only consider the binary shadow map itself, but also the general distortion which the Lorentz force brings about for particles of given rigidity in a given field. Rather than treating all trajectory endpoints which either end on the photosphere or at infinity as equivalent, we therefore first considered a background pattern, expressed as a function $I(\alpha, \delta)$ of longitudinal $(\alpha)$ and latitudinal $(\delta)$ position on the "celestial sphere" at infinity, being placed behind the magnetized region. The trajectory integrator thus maps a particle's starting direction $\left(\alpha_{0}, \delta_{0}\right)$ when crossing the viewing window to a final direction $\left(\alpha_{1}, \delta_{1}\right)$ at infinity, provided that the particle avoids the photosphere. This method allows us to characterize the distorting effect of magnetic deflection by imaging the distorted background $I\left(\alpha_{1}, \delta_{1}\right)$ as a function of the initial coordinates $\left(\alpha_{0}, \delta_{0}\right)$ in the viewing window. It has proven very useful to compare the result with expectations motivated by theoretical considerations, and was also instrumental to eliminate several coding errors from our numerical tools. Figure 2 illustrates selected examples of typical images thus obtained.

\section{Simulations vs. observations}

For actual simulations, a model of the actual coronal magnetic field is needed. We start from observed magnetograms (maps of the magnetic field components on the solar photosphere) available from the Global Oscillation Network Group (GONG, http://gong.nso.edu) for a given Carrington rotation, and assume that (i) field lines are purely radial at the so-called source surface, a Sun-centered sphere of radius $r=2.5$, and that (ii) the magnetic field in the intermediate region $r \in[1.0,2.5]$ assumes its lowest-energy state, which is that of a potential (current-free) field, $\vec{B}=-\nabla \Psi$. The scalar potential $\Psi$ may thus be obtained by solving the Laplace equation $\nabla^{2} \Psi=\nabla \cdot(\nabla \Psi)=-\nabla \cdot \vec{B}=0$, for which we use the FDIPS [8] tool. Beyond the source surface, field lines are continued in a divergence-free manner either with a simple radial $1 / r^{2}$ dependence or with the slightly more appropriate Parker spiral field [9].

Due to the fact that only sufficiently "straight" trajectories will produce a discernible shadow image and the very low CR flux at these correspondingly high energies, IceCube data will typically only allow for a single shadow image to be generated per season (covering about four months during the Antarctic summer). On the other hand, magnetic surface features such as active regions change on the somewhat shorter timescale of solar differential rotation of about 28 to 35 days (depending on heliolatitude). This implies that a single artificial shadow image has to be averaged over (i) several Carrington rotations and (ii) many (at least $~ 36$ ) different viewing angles during a given Carrington rotation. This requires the solar magnetic field to be be rotated around the $z$-axis, or, alternatively, the observer to revolve around this axis while keeping the static Sun's projection centered on the image plane. Both variants have been implemented and were shown to yield fully equivalent shadow images. Finally, the aforementioned averaging additionally has to be performed for various chemical elements, with the respective shadow images then averaged again, weighted by the observed elementary composition of the incident CR spectrum. Figure 3 shows respective sample shadow images for solar minimum and maximum conditions.

The most important quantity to be derived from the final shadow image is the shadow depth $s$, 


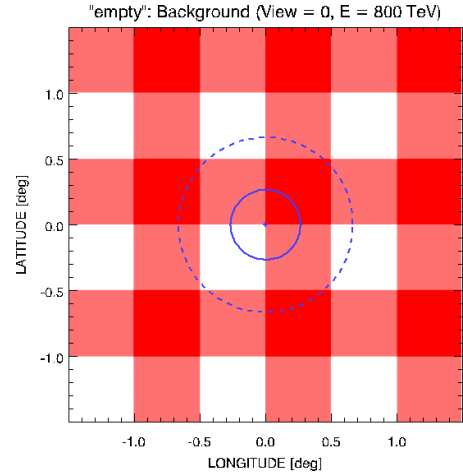

(A): no field

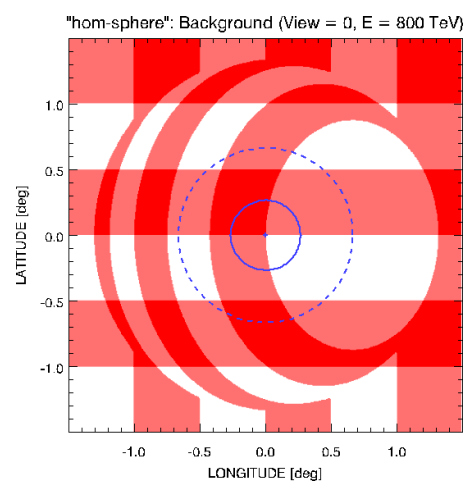

(D): homogeneous sphere

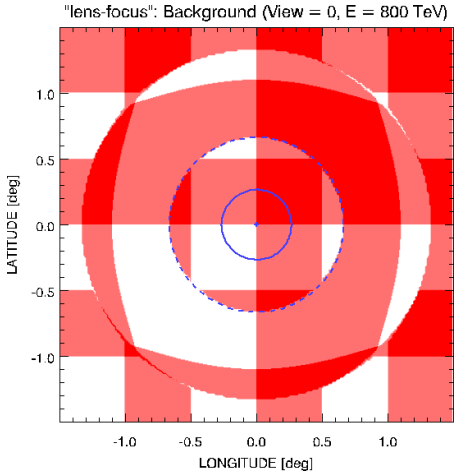

(B): focusing loop

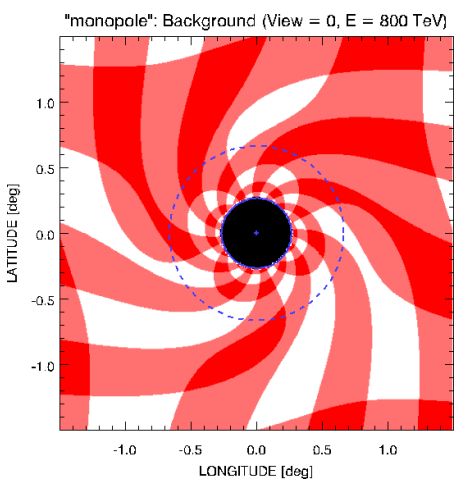

(E): monopole

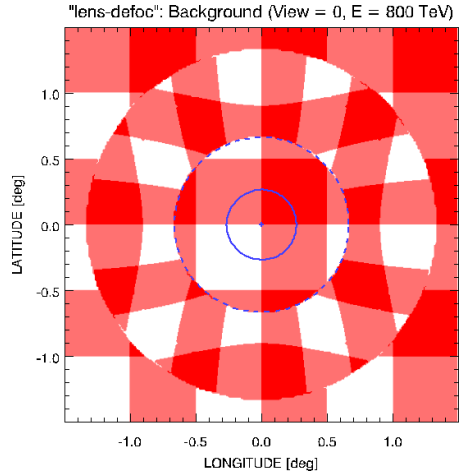

(C): defocusing loop

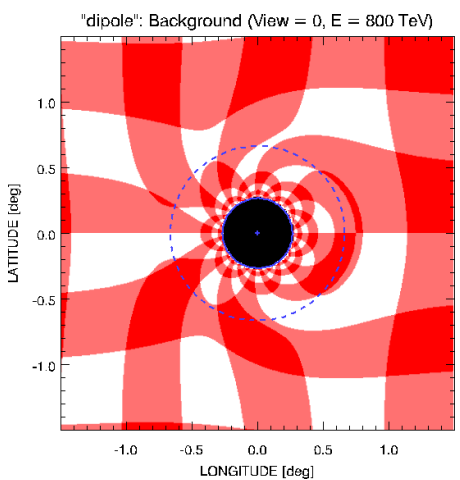

(F): point dipole

Figure 2: Test cases for magnetic ray tracing, using field configurations of increasing complexity in front of a checkered background pattern at infinity. A: unmagnetized reference case (no field at all); B: closed, ring-shaped field lines $\vec{B}=\left(z \vec{e}_{y}-y \vec{e}_{z}\right) / \sqrt{y^{2}+z^{2}}$ winding clockwise around the $x$-axis within a cylindrical annulus $\left(x, \sqrt{y^{2}+z^{2}}\right) \in[-0.2,+0.2] \times[2.5,5.0]$, creating a focusing lens with a pristine central region; $\mathrm{C}$ : the same configuration except with the field direction reversed, creating a defocusing effect; D: a homogeneous field $\vec{B}=0.1 \vec{e}_{z}$ confined to a sphere of radius 5 ; E: a monopole field $\vec{B}=\vec{r} / r^{3}$; and $\mathrm{F}$ : a $z$-aligned point dipole $\vec{B}=-\nabla\left(z / r^{3}\right)$. In the last two cases, trajectories are discontinued upon intersecting the unit sphere (corresponding to the solar photosphere), and the corresponding pixels are marked black. All test cases use a nominal proton $(Z=1)$ energy of $800 \mathrm{TeV}$ (back-tracing anti-protons) and magnetic field strength in units of $1 \mathrm{G}=0.1 \mathrm{mT}$. To guide the eye, blue lines mark circles of radii 1.0 (solid) and 2.5 (dashed).

defined via

$$
s:=\frac{\Delta \omega}{\pi\left(R_{\odot} / d_{0}\right)^{2}} \sum_{i=1}^{N}\left(1-I_{i}\right)
$$

as the amount by which the intensity $I_{i}$ of pixel $i$ differs from the full background intensity $\left(I_{i}=1\right)$, multiplied by the pixel's angular area $\Delta \omega$, summed over all $N \sim 100^{2}$ pixels, and then normalized to the size of the apparent solar disk. This quantity thus replicates the observational "relative deficit," with limiting cases of $s=1$ for purely geometrical shadowing by a Sun-sized opaque sphere, and $s=0$ for no shadowing taking place at all.

The $\eta$ scaling of Equations (2) and (3) suggests that for a given field configuration, the respective dependencies of $s$ on both $Z$ and $E$ can be condensed into one on rigidity $P \propto E / Z$. 

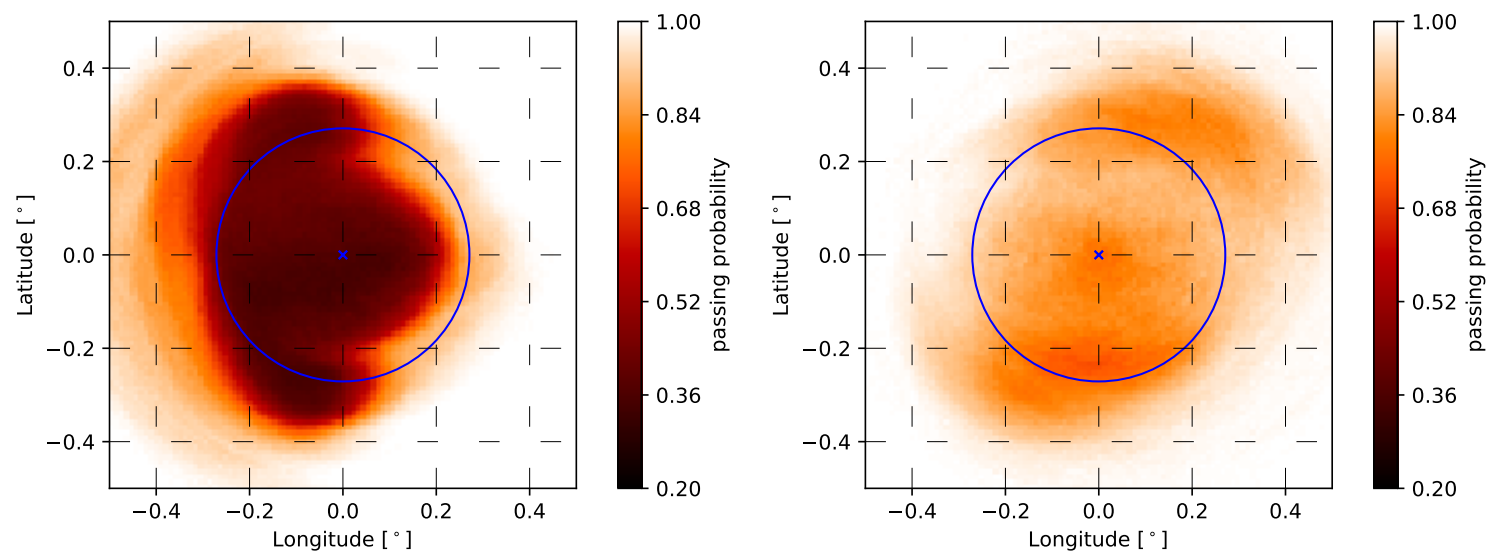

Figure 3: Examples of fully averaged shadow images (left: solar minimum of 2008/09; right: solar maximum of 2014/15) computed using the spectral CR composition according to [11]. Note the approximate equatorial mirror symmetry in the minimum case (indicative of a leading-order dipole field, see test case ' $F$ ' in Figure 2) compared to the approximate centrical symmetry in the maximum case (indicative of approximate leading-order radial symmetry, see test case ' $E$ ' in said figure). The pronounced difference in shadow depth is consistent with the trend shown in Figure 1. Taken from [10], wherein an extensive catalog of such computed images is presented.
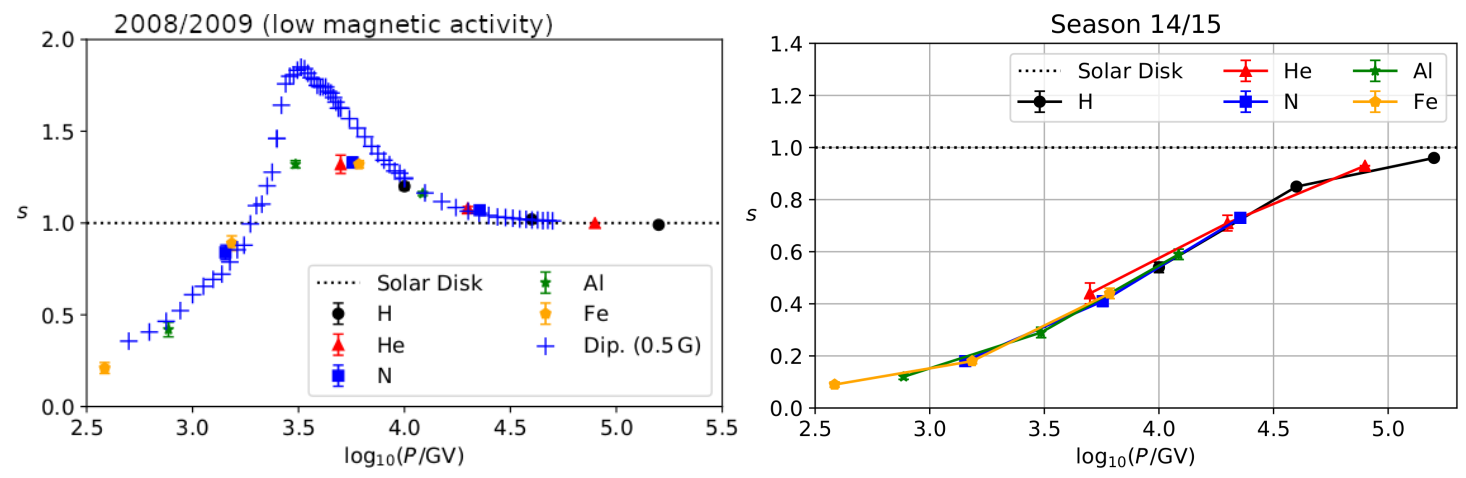

Figure 4: Sun shadow depth $s$ for $\mathrm{CR}$ particles of various elements as a function of rigidity, calculated for the 2008/09 (solar minimum, left) and 2014/15 (solar maximum, right) seasons. On the left, the total shadow depth resulting from a point dipole is overplotted. Each of these data points (blue crosses) corresponds to a shadow image, three of which are shown in Figure 5. Both datasets appear to agree best with a dimensionless dipolar moment of 0.5 , corresponding to a scaled field strength of $1 \mathrm{G}=0.1 \mathrm{mT}$ at the photospheric equator. Adapted from [10].

Indeed, Figure 4 shows that for a range of elements, the respective curves of $P \mapsto s(P)$ line up to form a single graph. It also shows that both the monotonous dependence and shadow-reducing effect of the coronal magnetic field $(s<1)$ reported previously by other groups is only found for the solar maximum season of 2014/15. During the 2008/09 solar minimum, however, a clear shadow maximum of nearly $s_{\max } \approx 1.3$ is apparent. This striking feature cannot be explained by the generally smaller coronal field strength, but is likely related to the very different field configuration prevailing during solar minimum, which more closely resembles a large-scale dipolar field.

In order to investigate this conjecture, we have run a sequence of simulations of mono-energetic 

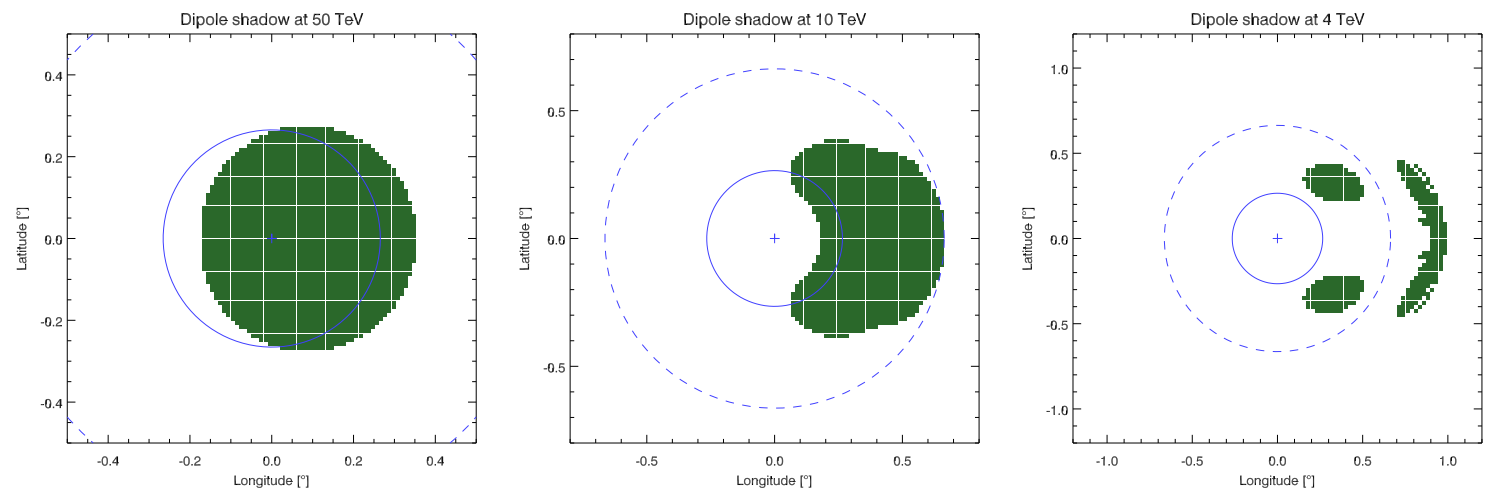

Figure 5: Shadow maps for a point dipole for proton energies of 50 (left), 10 (center), and $4 \mathrm{TeV}$ (right). Note the different fields of view relative to the solar photospheric disk (solid blue circle).

particles in a simple dipole as a crude model to the coronal magnetic field at times of solar minimum. Figure 5 shows three selected binary shadow images, indicating the expected disk-like morphology at high rigidities. At intermediate rigidities, the shadow area prominently exceeds that of the solar disk, while at still lower rigidities the shadow fragments into several symmetric "islands" before finally dissolving into the background. The shadow depth profile $P \mapsto s(P)$ resulting from a large number of such simulations has been overplotted on the left panel of Figure 4. As can clearly be seen here as well, we obtain the expected geometrical limit $s \rightarrow 1$ at very high energies, but then find a larger shadow near $10^{3.5} \mathrm{GV} \approx 3.2 \mathrm{TV}$ extending to $s_{\max } \approx 1.8$.

This latter comparison raises the question of which dipolar field strength, if any, should be considered "equivalent" to a given, more complex magnetic field in terms of its shadow-generating capabilities. We may tentatively argue that for a homogeneous magnetic field filling a finite region of space, the total deviation experienced by a traversing particle scales with (i) the magnitude of the homogeneous field and (ii) the length of its magnetized path. Computing, in this vein, the volume

integral $S:=\int_{r \in[1,2.5]}|\vec{B}| \mathrm{d} V$ for both the dipole and the potential field of Carrington rotation 2077 (December 2008) yields a ratio of $S_{\mathrm{CR} 2077} / S_{\text {dipole }} \approx 0.632$, in surprisingly good agreement with the ad hoc best-fit value of 0.5 used in the comparison shown in the left panel of Figure 4.

\section{Conclusions}

We have presented and analyzed artificial images of the Sun's cosmic-ray shadow generated by back-tracing of virtual anti-particles in a coronal magnetic field which derives from photospheric boundary conditions in the absence of electric currents. We could confirm the trend previously found in both simulation and observation according to which high solar activity correlates with weak (sub-geometric) shadows, but mostly so for solar maximum conditions. The unexpected occurrence of stronger-than-geometric shadows during solar minimum and its non-monotonous dependence on rigidity could successfully be linked to the effect of a large-scale dipolar magnetic field. Although more sophisticated simulations (as reported in, e.g. [4]) are available, it is the relative simplicity of this dipolar case that makes it an ideal demonstrator of this novel effect.

While attempts to directly constrain the coronal magnetic field through Sun shadow observations are forestalled by the unfortunate discrepancy between observational and solar dynamic 
timescales, the presented investigations serve to strengthen this potentially fruitful link between solar physics and high-energy cosmic rays, to be further explored in forthcoming investigations.

\section{Acknowledgements}

The presenting author acknowledges financial support through the Ruhr Astroparticle and Plasma Physics (RAPP) Center (MERCUR project St-2014-040) and the German Research Foundation (Deutsche Forschungsgemeinschaft, DFG) through projects FI 706/27-1 and TJ 62/7-1.

\section{References}

[1] M. Amenomori, Z. Cao, L.K. Ding, Z.Y. Feng, K. Hibino, N. Hotta et al., Direct Evidence of the Interplanetary Magnetic Field Effect on the Cosmic-Ray Shadow by the Sun, Astrophys. J. Lett. 415 (1993) L147.

[2] A. Albert, M. André, M. Anghinolfi, G. Anton, M. Ardid, J.J. Aubert et al., Observation of the cosmic ray shadow of the Sun with the ANTARES neutrino telescope, Phys. Rev. D 102 (2020) 122007 [2007.00931].

[3] M.G. Aartsen, M. Ackermann, J. Adams, J.A. Aguilar, M. Ahlers, M. Ahrens et al., Detection of the Temporal Variation of the Sun's Cosmic Ray Shadow with the IceCube Detector, Astrophys. J. 872 (2019) 133 [1811.02015].

[4] M.G. Aartsen, R. Abbasi, M. Ackermann, J. Adams, J.A. Aguilar, M. Ahlers et al., Measurements of the time-dependent cosmic-ray Sun shadow with seven years of IceCube data: Comparison with the Solar cycle and magnetic field models, Phys. Rev. D 103 (2021) 042005 [2006. 16298].

[5] J.P. Boris, Relativistic plasma simulation-optimization of a hybrid code, in Proc. of the 4th Conf. on Numerical Simulation of Plasmas, (Washington, D.C.), pp. 3-67, Naval Res. Lab., NRL, Nov, 1970.

[6] J. Stam and E. Languénou, Ray tracing in non-constant media, in Eurographics Workshop on Rendering Techniques, pp. 225-234, 01, 1996, DOI.

[7] E. Gröller, Nonlinear ray tracing: Visualizing strange worlds, Vis. Comput. 11 (1995) 263.

[8] G. Tóth, B. van der Holst and Z. Huang, Obtaining Potential Field Solutions with Spherical Harmonics and Finite Differences, Astrophys. J. 732 (2011) 102 [1104 . 5672].

[9] E.N. Parker, Dynamics of the Interplanetary Gas and Magnetic Fields., Astrophys. J. 128 (1958) 664.

[10] J. Becker Tjus, P. Desiati, N. Döpper, H. Fichtner, J. Kleimann, M. Kroll et al., Cosmic-ray propagation around the Sun: investigating the influence of the solar magnetic field on the cosmic-ray Sun shadow, Astron. Astrophys. 633 (2020) A83 [1903.12638].

[11] T.K. Gaisser and M. Honda, Flux of atmospheric neutrinos, Annu. Rev. Nucl. Part. Sci. 52 (2002) 153 [hep-ph/0203272]. 